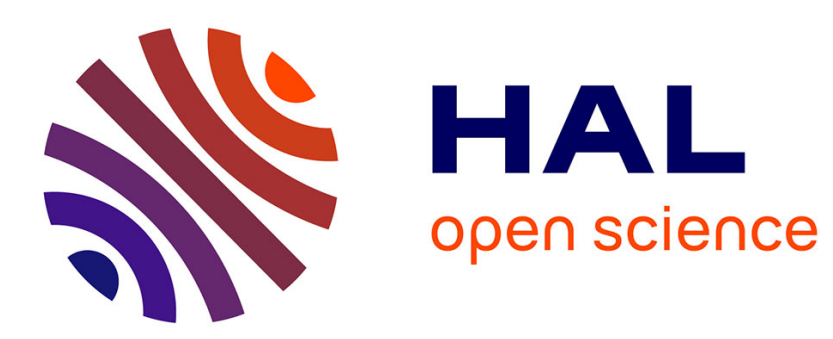

\title{
Self-contact and fictitious domain using a difference convex approach
}

\author{
Stéphane Pagano, Pierre Alart
}

\section{To cite this version:}

Stéphane Pagano, Pierre Alart. Self-contact and fictitious domain using a difference convex approach. International Journal for Numerical Methods in Engineering, 2008, 75, pp.29-42. 10.1002/nme.2241 . hal-00347455

\section{HAL Id: hal-00347455 \\ https://hal.science/hal-00347455}

Submitted on 16 Dec 2008

HAL is a multi-disciplinary open access archive for the deposit and dissemination of scientific research documents, whether they are published or not. The documents may come from teaching and research institutions in France or abroad, or from public or private research centers.
L'archive ouverte pluridisciplinaire HAL, est destinée au dépôt et à la diffusion de documents scientifiques de niveau recherche, publiés ou non, émanant des établissements d'enseignement et de recherche français ou étrangers, des laboratoires publics ou privés. 


\title{
Self-contact and fictitious domain using a difference convex approach
}

\author{
S. Pagano*and P. Alart \\ Laboratoire de Mécanique et Génie Civil, \\ Université Montpellier II - CNRS UMR 5508 \\ Place Eugene Bataillon, 34095 Montpellier Cedex, France
}

\begin{abstract}
SUMMARY
A numerical approach of contact or self-contact of thin structures is performed using a relaxed orientation-preserving condition inserted in the difference of convex function framework. This method is analyzed on academic examples and cellular media. Copyright (c) 2000 John Wiley \& Sons, Ltd.
\end{abstract}

KEY WORDS: Difference of convex function; Self-contact; Fictitious domains

\section{Self contact and fictitious domain}

Contact is generally formalized as a local condition between points or surfaces previously identified as opposite entities. This is a classic approach in finite element modelling and provides specific joint or contact elements [14]. It is easy to implement in the context of small perturbations whose joint elements do not to need redefining during the process. In finite deformation the implementation is more technical; it needs to modify the mesh of joint elements at certain steps of an incremental process. In the case of hyperelasticity with frictionless contact, an incremental procedure is nevertheless necessary to identify the potential contact elements (unless one defines the boundary as the potential contact surface - it is obviously unwieldy). To avoid having to identify contact elements, global criteria can be used that are restricted to characterizing self-contact to determine the finite deformation of a single connex domain. Self-contact is related to the injectivity property, whose local version is called the orientation-preserving condition and involves the determinant of the gradient of deformation $\Psi$ over a domain $\Omega_{f}$,

$$
\operatorname{det} \nabla \Psi(\mathbf{x})>0
$$

almost everywhere in $\Omega_{f}$. However the minimizing solution of the associated minimization problem is globally injective if a global condition is also assured (i.e. in addition to (1)). For a

\footnotetext{
* Correspondence to: LMGC, Université Montpellier II - CNRS UMR 5508

Place Eugene Bataillon, 34005 Montpellier Cedex, France

Contract/grant sponsor: ; contract/grant number:
} 
pure displacement problem, i.e. with a prescribed displacement field on the whole boundary, it is necessary to assume the existence of globally injective mapping satisfying the imposed displacement over the whole boundary (cf. [5], theorems 1 and 4).

An enlightening counter-example given by Ball [5] shows that the global condition cannot be omitted. Let the mapping $\phi$ of the unit disc $\Omega_{f}$ in $\mathbb{R}^{2}$ be defined in polar coordinates $(r, \theta)$ by,

$$
\phi:(r, \theta) \rightarrow\left(\frac{1}{\sqrt{2}} r, 2 \theta\right)
$$

It is easy to check that $\operatorname{det} \nabla \phi(\mathbf{x})=1$ if $\mathbf{x} \neq 0$, but $\phi$ is not locally invertible at the origin.

For traction-displacement problems Ciarlet [7] proposes another global injectivity condition to the admissible deformations. But this condition is also a mathematical model of self-contact without friction for a smooth energy minimizer. The admissible deformations $\Psi$ have to satisfy the following additional global condition over the whole domain,

$$
\int_{\Omega_{f}} \operatorname{det} \nabla \Psi d \mathbf{x} \leq \operatorname{vol} \Psi\left(\Omega_{f}\right) .
$$

Without this global assumption, the interpenetration cannot be numerically ruled out as shown in the following example. Consider for instance the mapping

$$
\phi: \mathbf{x} \in \Omega \subset \mathbb{R}^{3} \rightarrow\left(x_{1} \cos \frac{x_{2}}{l}, x_{1} \sin \frac{x_{2}}{l}, x_{3}\right),
$$

where $\Omega$ is a rectangular rod of length $2 \theta l$ parallel to the vector $e_{2}$ as shown in Figure 1. Then $\operatorname{det} \nabla \phi(\mathbf{x})=x_{1} / l$ for all $\mathbf{x} \in \Omega$, yet for $\theta \geq \pi$ the mapping is not injective since $\phi\left(x_{1}, \pi l, x_{3}\right)=\phi\left(x_{1},-\pi l, x_{3}\right)$. For $\theta=\pi$ the injectivity is lost on the boundary, while for $\theta>\pi$, interpenetration has occurred. In this case,

$$
\int_{\Omega_{f}} \operatorname{det} \nabla \Psi d \mathbf{x}=\theta\left(d_{2}^{2}-d_{1}^{2}\right),
$$

and

$$
\operatorname{vol} \Psi\left(\Omega_{f}\right)=\pi\left(d_{2}^{2}-d_{1}^{2}\right) .
$$

The condition (3) is not satisfied for $\theta>\pi$.

Unfortunately these global conditions are not easy to handle in numerical treatment. Even the local orientation-preserving condition is not accounted for in finite element codes; it is only verified a posteriori. Note that a similar constraint has to be numerically treated for incompressible materials [9]: the determinant of the deformation gradient is equal to one almost everywhere, and not only positive. In this paper, we propose an alternative numerical strategy which lacks a firm theoretical background, but is convenient for a friendly implementation. In some situations interpenetration can be excluded by imposing an extended orientationpreserving condition to a well-chosen fictitious domain. For example in Figure 2 the fictitious domain may be limited to the air-gap according to the imposed loading. A cellular material is a peculiarly well-adapted case for which the choice is very straightforward i.e. the interior of each cell. This honeycomb structure, viewed as a truss of flexible rods, will be the final application of this work. The strict inequality (1), which does not allow self-contact, is replaced by a non negative inequality,

$$
\operatorname{det} \nabla \Psi(\mathbf{x}) \geq 0,
$$




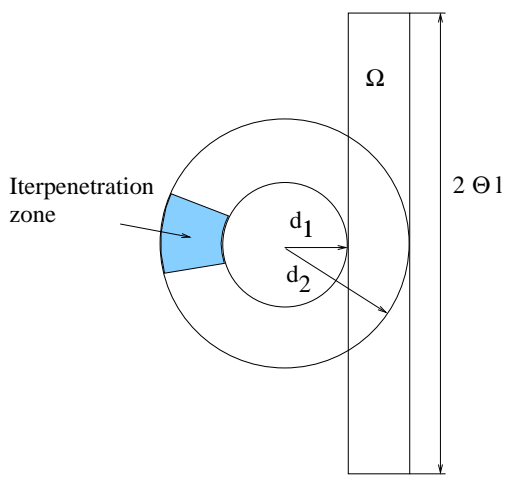

Figure 1. An orientation-preserving $\phi$ that is not globally injective.
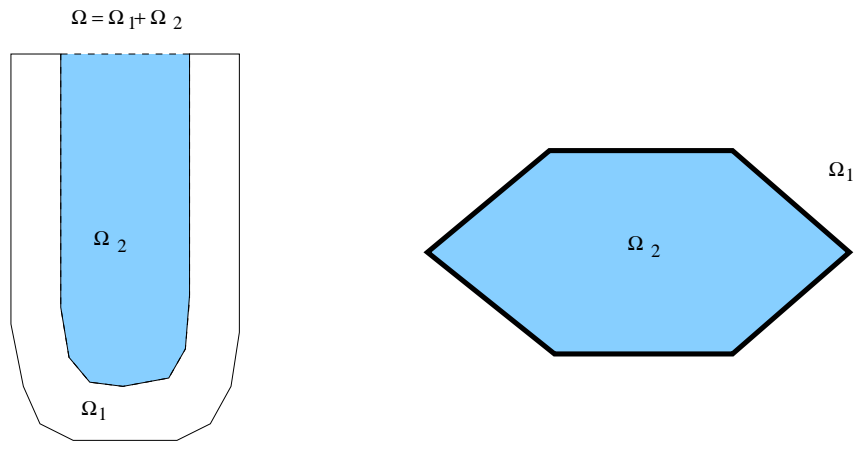

Figure 2. Two examples of fictitious domains.

almost everywhere in $\Omega_{f}$. Moreover the bulk energy of the fictitious domain is assumed to be null. The D.C. approach (for difference of convex functions) has already been applied to reach local minima of non convex problems in material sciences $[12,11]$ or in structural mechanics with post-buckling $[2,3]$. It may be viewed as a general non convex solver. However in $[2,3]$ the confinement is performed using a contact condition between the deformable body and the obstacles, which is treated by an additional Newton solver [1]. In the present work the (possibly self-) contact condition is replaced by the relaxed orientation-preserving constraint (4) that is strongly non linear but may be formulated using D.C. splitting. De Saxce [8] starts from a linearization of (4) and derives a classical linear complementary problem (LCP).

Section 2 details a common D.C. formulation for two constraints: the inextensibility condition of flexible rods similar to the incompressibility for three-dimensional bodies and the self-contact condition by the way of fictitious domain injectivity (4). Section 3 is devoted to the development of a D.C. solver coupling self-contact and inextensibility with detailed implementation aspects. Section 4 provides some numerical simulations applied to cellular structures. 
For a simplified presentation we consider two thin rods of length $\ell$ whose deformed configurations take place in a $s-t$ plane and the rectangular fictitious domain $\Omega_{f}=(0, \ell) \times(0, e)$ is included between the two rods (cf. Figure 3). We denote by $\Gamma_{N}$ and $\Gamma_{S}$ the upper and lower boundaries of $\Omega_{f}$. The configuration is described by a vector function $\mathbf{y}$ depending on $s$ and $t$. We restrict ourselves here to the case of inextensible rods $[2,3]$. The inextensibility condition may be written as an equality constraint of the configuration function for each of the two rods,

$$
h\left(\partial_{s} \mathbf{y}(s, 0)\right)=h\left(\partial_{s} \mathbf{y}(s, e)\right)=0
$$

where $h(\mathbf{x})=\|\mathbf{x}\|^{2}-1$

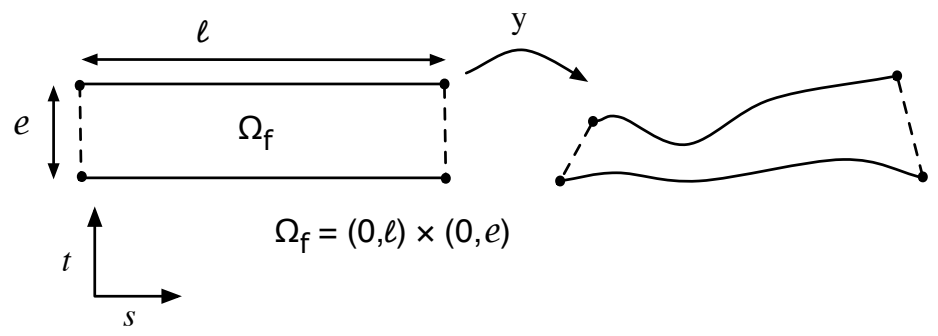

Figure 3. Domain $\Omega_{f}$ before and after deformation

This assumption leads to consider only the bending term in the expression of bulk energy. The contact between the two rods (identified to the boundaries $\Gamma_{N}$ and $\Gamma_{S}$ ) is taken into account by the relaxed orientation-preserving condition (4) applied to the fictitious domain $\Omega_{f}$. If the two rods are submitted to loading fields $\mathbf{f}_{0}$ and $\mathbf{f}_{e}$, the potential energy takes the simplified form,

$$
\begin{aligned}
J(\mathbf{y})= & \frac{1}{2} \int_{0}^{\ell} E(s, 0) I(s, 0)\left\|\partial_{s}^{2} \mathbf{y}(s, 0)\right\|^{2} d s+\frac{1}{2} \int_{0}^{\ell} E(s, e) I(s, e)\left\|\partial_{s}^{2} \mathbf{y}(s, e)\right\|^{2} d s \\
& -\int_{0}^{\ell} \mathbf{f}_{0}(s) \cdot \mathbf{y}(s, 0) d s-\int_{0}^{\ell} \mathbf{f}_{e}(s) \cdot \mathbf{y}(s, e) d s,
\end{aligned}
$$

where $E$ is the Young modulus and $I$ is the geometrical inertia of the section (i.e., $E I$ is the (positive) bending stiffness) which may depend on the abscissa $s$. Consequently an equilibrium state may be characterized as a local minimum of the potential energy restricted to the kinematically admissible configurations:

$$
\inf _{\mathbf{y} \in \mathcal{A} \cap \mathcal{C} \cap \mathcal{I}}(l o c) J(\mathbf{y})
$$

The set of admissible configurations is the intersection of three sets, the first containing the boundary conditions, the second accounting for the inextensibility condition and the last 
imposing the relaxed orientation-preserving condition:

$$
\begin{aligned}
\mathrm{H} & =\left\{\mathbf{y} \in \mathrm{H}^{1}(\Omega) ;(\mathbf{y}(., 0), \mathbf{y}(., e)) \in \mathrm{H}^{2}(0, \ell)\right\} \\
\mathcal{A} & =\{\mathbf{y} \in \mathrm{H} ; \text { plus adequate boundary conditions }\} \\
\mathcal{C} & =\left\{\mathbf{y} \in \mathrm{H} ; h\left(\partial_{s} \mathbf{y}(s, 0)\right)=0 \text { and } h\left(\partial_{s} \mathbf{y}(s, e)\right)=0 \text { a.e. } s \in\right] 0, \ell[\} . \\
\mathcal{I} & =\{\mathbf{y} \in \mathrm{H} ; \operatorname{det}(\nabla \mathbf{y}(s, t)) \geq 0 \text { a.e. }(s, t) \in \Omega\} .
\end{aligned}
$$

Since the objective function $J$ is convex, non convexity is due to the non convexity of the two sets $\mathcal{C}$ and $\mathcal{I}$.

\section{D.C. algorithm and implementation aspects}

Using the formulae (6) and (7) we introduce an arbitrary admissible configuration $\overline{\mathbf{y}}$ so that the displacement field $\mathbf{v}=\mathbf{y}-\overline{\mathbf{y}}$ belongs to the vector sub-space $\mathcal{V}$ associated with $\mathcal{A}$. We then define three affine differential operators $D^{S}, D^{N}, \mathbf{D}$, such that

$$
\begin{aligned}
D^{S} \mathbf{v}(s) & =\left(\partial_{s} \overline{\mathbf{y}}+\partial_{s} \mathbf{v}\right)(s, 0), \\
D^{N} \mathbf{v}(s) & =\left(\partial_{s} \overline{\mathbf{y}}+\partial_{s} \mathbf{v}\right)(s, e), \\
\mathbf{D} \mathbf{v}(s, t) & =(\nabla \overline{\mathbf{y}}+\nabla \mathbf{v})(s, t) .
\end{aligned}
$$

In this way, the relevant potential energy (6) is expressed as a function $\varphi(\mathbf{v})$ of $\mathbf{v}$ :

$$
\begin{aligned}
J(\mathbf{y})=\varphi(\mathbf{v})= & \int_{0}^{\ell} \frac{E I(s, 0)}{2}\left\|\left(\partial_{s}^{2} \overline{\mathbf{y}}+\partial_{s}^{2} \mathbf{v}\right)(s, 0)\right\|^{2} d s+\int_{0}^{\ell} \frac{E I(s, e)}{2}\left\|\left(\partial_{s}^{2} \overline{\mathbf{y}}+\partial_{s}^{2} \mathbf{v}\right)(s, e)\right\|^{2} d s \\
& -\int_{0}^{\ell} \mathbf{f}_{0}(s) \cdot \mathbf{v}(s, 0) d s-\int_{0}^{\ell} \mathbf{f}_{e}(s) \cdot \mathbf{v}(s, e) d s .
\end{aligned}
$$

The local minimization problem is then formulated as a constrained problem on the $\mathbf{v}$ field,

$$
\inf _{\mathbf{y} \in \mathcal{A} \cap \mathcal{C} \cap \mathcal{I}}(l o c) J(\mathbf{y}) \Longleftrightarrow \quad \inf _{\mathbf{v} \in \mathcal{V}, h\left(D^{S} \mathbf{v}\right)=0, h\left(D^{N} \mathbf{v}\right)=0, \operatorname{det}(\mathbf{D v}) \geq 0}(l o c) \varphi(\mathbf{v}) .
$$

Starting from (16) and introducing three scalar multiplier fields $\mu_{S}, \mu_{N}$ and $\nu$, we define a saddle-point problem for which the Lagrangian function is not convex with respect to the first variable $\mathbf{v}$ and linear with respect to three Lagrange multipliers,

$$
\inf _{\mathbf{v} \in \mathcal{V}}(l o c) \sup _{\mu_{S}, \mu_{N} \in \mathrm{L}^{2}(0, \ell), \nu \in \mathrm{L}^{2}(\Omega) s . t . \nu \leq 0} L\left(\mathbf{v} ; \mu_{S}, \mu_{N}, \nu\right),
$$

where

$$
\begin{aligned}
L\left(\mathbf{v} ; \mu_{S}, \mu_{N}, \nu\right)= & \varphi(\mathbf{v})+\int_{0}^{\ell} \mu_{S}(s) h\left(D^{S} \mathbf{v}(s)\right) d s+\int_{0}^{\ell} \mu_{N}(s) h\left(D^{N} \mathbf{v}(s)\right) d s \\
& +\int_{0}^{\ell} \int_{0}^{e} \nu(s, t) \operatorname{det}(\mathbf{D v}(s, t)) d t d s .
\end{aligned}
$$

A stationary point $\left(\mathbf{u}, \lambda_{S}, \lambda_{N}, \gamma\right)$ of the Lagrangian functional is the solution of the EulerLagrange equations,

$$
\begin{aligned}
& 0=\frac{\partial L}{\partial \mathbf{v}}\left(\mathbf{u} ; \lambda_{S}, \lambda_{N}, \gamma\right), \\
& 0=\frac{\partial L}{\partial \boldsymbol{\mu}_{S}}\left(\mathbf{u} ; \lambda_{S}, \lambda_{N}, \gamma\right), \quad 0=\frac{\partial L}{\partial \boldsymbol{\mu}_{N}}\left(\mathbf{u} ; \lambda_{S}, \lambda_{N}, \gamma\right), \quad 0=\frac{\partial L}{\partial \nu}\left(\mathbf{u} ; \lambda_{S}, \lambda_{N}, \gamma\right)
\end{aligned}
$$


To deal with the first equation (19) we introduce the following splitting of the Lagrangian into the difference of two convex functions [3],

$$
L\left(\mathbf{v} ; \mu_{S}, \mu_{N}, \nu\right)=\Phi_{1}\left(\mathbf{v} ; \mu_{S}, \mu_{N}, \nu\right)-\Phi_{2}\left(\mathbf{v} ; \mu_{S}, \mu_{N}, \nu\right),
$$

where,

$$
\begin{aligned}
\Phi_{1}\left(\mathbf{v} ; \mu_{S}, \mu_{N}, \nu\right)= & \varphi(\mathbf{v})+\int_{0}^{\ell} \mu_{S}^{+}(s) h\left(D^{S} \mathbf{v}(s)\right) d s+\int_{0}^{\ell} \mu_{N}^{+}(s) h\left(D_{N} \mathbf{v}(s)\right) d s \\
& -\int_{0}^{\ell} \int_{0}^{e} \nu(s, t) \operatorname{det}_{1}(\mathbf{D v}(s, t)) d s d t \\
\Phi_{2}\left(\mathbf{v} ; \mu_{S}, \mu_{N}, \nu\right)= & \int_{0}^{\ell} \mu_{S}^{-}(s) h\left(D^{S} \mathbf{v}(s)\right) d s+\int_{0}^{\ell} \mu_{N}^{-}(s) h\left(D^{N} \mathbf{v}(s)\right) d s \\
& -\int_{0}^{\ell} \int_{0}^{e} \nu(s, t) \operatorname{det}_{2}(\mathbf{D v}(s, t)) d s d t \\
= & \Phi_{2}^{S}\left(D^{S} \mathbf{v} ; \mu_{S}\right)+\Phi_{2}^{N}\left(D^{N} \mathbf{v} ; \mu_{N}\right)+\Phi_{2}^{\nabla}(\mathbf{D v} ; \nu) .
\end{aligned}
$$

The functions $\mu^{-}$and $\mu^{+}$already used in [3] are defined as follow,

$$
\mu_{i}^{+}=\max \left(0, \mu_{i}\right), \quad \mu_{i}^{-}=\max \left(0,-\mu_{i}\right), i=N, S .
$$

In a bi-dimensional case, the determinant of a matrix can be split into the difference of two convex functions $\operatorname{det}_{1}$ and $\operatorname{det}_{2}$,

$$
\operatorname{det}(\mathbf{C})=\operatorname{det}_{2}(\mathbf{C})-\operatorname{det}_{1}(\mathbf{C}),
$$

where

$$
\begin{aligned}
\operatorname{det}_{1}(\mathbf{C}) & =\frac{1}{2}\left[\left(C_{12}+C_{21}\right)^{2}+C_{11}^{2}+C_{22}^{2}\right] . \\
\operatorname{det}_{2}(\mathbf{C}) & =\frac{1}{2}\left[\left(C_{11}+C_{22}\right)^{2}+C_{12}^{2}+C_{21}^{2}\right],
\end{aligned}
$$

As previously introduced in $[4,2,3,13]$, it is straightforward to define a type II Lagrangian depending on seven fields by using the Fenchel transform of the function $\Phi_{2}$ with respect to the first variable $\mathbf{v}$,

$$
\begin{aligned}
L\left(\mathbf{v} ; \mu_{S}, \mu_{N}, \nu\right)= & \Phi_{1}\left(\mathbf{v} ; \mu_{S}, \mu_{N}, \nu\right)-\Phi_{2}^{S}\left(D^{S} \mathbf{v} ; \mu_{S}\right)-\Phi_{2}^{N}\left(D^{N} \mathbf{v} ; \mu_{N}\right)-\Phi_{2}^{\nabla}(\mathbf{D v} ; \nu), \\
= & \Phi_{1}(\mathbf{v} ; \mu)-\sup _{\boldsymbol{\tau}_{S}, \boldsymbol{\tau}_{N}, \boldsymbol{\tau}_{\nabla}}\left\{\int_{0}^{\ell} D^{S} \mathbf{v} \cdot \boldsymbol{\tau}_{S} d s+\int_{0}^{\ell} D^{N} \mathbf{v} \cdot \boldsymbol{\tau}_{N} d s\right. \\
& \left.+\int_{0}^{\ell} \int_{0}^{e} \mathbf{D v}: \boldsymbol{\tau}_{\nabla} d s d t-\Phi_{2}^{S *}\left(\boldsymbol{\tau}_{S} ; \mu_{S}\right)-\Phi_{2}^{N *}\left(\boldsymbol{\tau}_{N} ; \mu_{N}\right)-\Phi_{2}^{\nabla *}\left(\boldsymbol{\tau}_{\nabla} ; \nu\right)\right\}, \\
= & \inf _{S}, \boldsymbol{\tau}_{N}, \boldsymbol{\tau}_{\nabla} L_{\mathbb{I}}\left(\mathbf{v}, \boldsymbol{\tau}_{S}, \boldsymbol{\tau}_{N}, \boldsymbol{\tau}_{\nabla} ; \mu_{S}, \mu_{N}, \nu\right),
\end{aligned}
$$

Copyright (c) 2000 John Wiley \& Sons, Ltd.

Int. J. Numer. Meth. Engng 2000; 00:1-6 
where

$$
\begin{array}{r}
\mathrm{L}_{\mathbb{I}}\left(\mathbf{v}, \boldsymbol{\tau}_{S}, \boldsymbol{\tau}_{N}, \boldsymbol{\tau}_{\nabla} ; \mu_{S}, \mu_{N}, \nu\right)=\Phi_{1}\left(\mathbf{v} ; \mu_{S}, \mu_{N}, \nu\right)+\Phi_{2}^{S *}\left(\boldsymbol{\tau}_{S} ; \mu_{S}\right)+\Phi_{2}^{N *}\left(\boldsymbol{\tau}_{N} ; \mu_{N}\right) \\
+\Phi_{2}^{\nabla *}\left(\boldsymbol{\tau}_{\nabla} ; \nu\right)-\int_{0}^{\ell} D^{S} \mathbf{v} \cdot \boldsymbol{\tau}_{S} d s-\int_{0}^{\ell} D^{N} \mathbf{v} \cdot \boldsymbol{\tau}_{N} d s-\int_{0}^{\ell} \int_{0}^{e} \mathbf{D v}: \boldsymbol{\tau}_{\nabla} d t d s .
\end{array}
$$

A "saddle point" type problem may be associated to the first Lagrangian $L$,

Find $\left(\mathbf{u}, \boldsymbol{\sigma}_{S}, \boldsymbol{\sigma}_{N}, \boldsymbol{\sigma}_{\nabla} ; \lambda_{S}, \lambda_{N}, \gamma\right) \in \mathcal{H}$ such that

$$
\begin{aligned}
\mathrm{L}_{\mathbb{I}}\left(\mathbf{u}, \boldsymbol{\sigma}_{S}, \boldsymbol{\sigma}_{N}, \boldsymbol{\sigma}_{\nabla} ; \mu_{S}, \mu_{N}, \nu\right) \leq \mathrm{L}_{\mathbb{I}}\left(\mathbf{v}, \boldsymbol{\sigma}_{S}, \boldsymbol{\sigma}_{N}, \boldsymbol{\sigma}_{\nabla} ; \lambda_{S}, \lambda_{N}, \gamma\right) \\
\quad \leq \mathrm{L}_{\mathbb{I}}\left(\mathbf{v}, \boldsymbol{\tau}_{S}, \boldsymbol{\tau}_{N}, \boldsymbol{\tau}_{\nabla} ; \lambda_{S}, \lambda_{N}, \gamma\right), \quad \forall\left(\mathbf{v}, \boldsymbol{\tau}_{S}, \boldsymbol{\tau}_{N}, \boldsymbol{\tau}_{\nabla} ; \mu_{S}, \mu_{N}, \nu\right) \in \mathcal{H},
\end{aligned}
$$

where $\mathcal{H}=\mathcal{V} \times\left(\mathrm{L}^{2}(0, \ell)\right)^{2} \times \mathrm{L}^{2}(\Omega) \times\left(\mathrm{L}^{2}(0, \ell)\right)^{2} \times \mathrm{L}^{2}(\Omega)$.

Naturally, we can derive different solution methods from this formulation, based on the Uzawa algorithm to solve the equations $(19,20)$. Our choice was to fully solve the equation (19) by a D.C. algorithm before updating the Lagrange multipliers according to the Uzawa iteration (20), defining the DCalg1 method ( cf. table I ). We modified the updating to account for the negative condition on $\gamma$. The D.C. algorithm, as introduced by Auchmuty [4], consists in successively minimizing the type II Lagrangian $\mathrm{L}_{\mathrm{II}}$ with respect to each variable $\mathbf{v}, \boldsymbol{\tau}_{S}, \boldsymbol{\tau}_{N}, \boldsymbol{\tau}_{\nabla}$ as specified in the four sub steps a, b, c and d of step 1: indeed, the first two steps deal with the inextensibility condition and the last two with the orientation-preserving condition. In step 2 we first updated the multipliers associated with the inextensibility condition and finished with that associated with the orientation-preserving condition.

Table I. DCalg1 algorithm.

- Initialization of the algorithm with $\left(\mathbf{u}^{0}, \boldsymbol{\sigma}_{S}^{0}, \boldsymbol{\sigma}_{N}^{0}, \boldsymbol{\sigma}_{\nabla}^{0} ; \lambda_{S}^{0}, \lambda_{N}^{0}, \gamma^{0}\right)$,

- $\left(\mathbf{u}^{n-1}, \boldsymbol{\sigma}_{S}^{n-1}, \boldsymbol{\sigma}_{N}^{n-1}, \boldsymbol{\sigma}_{\nabla}^{n-1} ; \lambda_{S}^{n-1}, \lambda_{N}^{n-1}, \gamma^{n-1}\right)$ known, determine $\mathbf{u}^{n}, \boldsymbol{\sigma}_{S}^{n}, \boldsymbol{\sigma}_{N}^{n}, \boldsymbol{\sigma}_{\nabla}^{n} ; \lambda_{S}^{n}, \lambda_{N}^{n}, \gamma^{n}$ as follows,

step 1 : Determination of $\left(\mathbf{u}^{n}, \boldsymbol{\sigma}_{S}^{n}, \boldsymbol{\sigma}_{N}^{n}, \boldsymbol{\sigma}_{\nabla}^{n}\right)$ by the D.C. algorithm on $\mathrm{L}_{\mathbb{I}}\left(., ., ., . ; \lambda_{S}^{n-1}, \lambda_{N}^{n-1}, \gamma^{n-1}\right)$

$$
\begin{array}{ll}
\text { a : } & \mathbf{u}^{n, i}=\arg \min \mathrm{L}_{\mathbb{I}}\left(., \boldsymbol{\sigma}_{S}^{n, i-1}, \boldsymbol{\sigma}_{N}^{n, i-1}, \boldsymbol{\sigma}_{\nabla}^{n, i-1} ; \lambda_{S}^{n-1}, \lambda_{N}^{n-1}, \gamma^{n-1}\right) \\
\mathrm{b}: & \boldsymbol{\sigma}_{S}^{n, i} \in \arg \min \mathrm{L}_{\mathbb{I}}\left(\mathbf{u}^{n, i}, ., \boldsymbol{\sigma}_{N}^{n, i-1}, \boldsymbol{\sigma}_{\nabla}^{n, i-1} ; \lambda_{S}^{n-1}, \lambda_{N}^{n-1}, \gamma^{n-1}\right) \\
\mathrm{c}: & \boldsymbol{\sigma}_{N}^{n, i} \in \arg \min \mathrm{L}_{\mathbb{I}}\left(\mathbf{u}^{n, i}, \boldsymbol{\sigma}_{S}^{n, i}, ., \boldsymbol{\sigma}_{\nabla}^{n, i-1} ; \lambda_{S}^{n-1}, \lambda_{N}^{n-1}, \gamma^{n-1}\right) \\
\mathrm{d}: & \boldsymbol{\sigma}_{\nabla}^{n, i} \in \arg \min \mathrm{L}_{\mathbb{I}}\left(\mathbf{u}^{n, i}, \boldsymbol{\sigma}_{S}^{n, i}, \boldsymbol{\sigma}_{N}^{n, i}, ; \lambda_{S}^{n-1}, \lambda_{N}^{n-1}, \gamma^{n-1}\right)
\end{array}
$$

step 2 : Updating of the multiplier :

$$
\begin{aligned}
& \lambda_{S}^{n}=\lambda_{S}^{n-1}+\rho h\left(D^{S} \mathbf{u}^{n}\right) \\
& \lambda_{N}^{n}=\lambda_{N}^{n-1}+\rho h\left(D^{N} \mathbf{u}^{n}\right) \\
& \gamma^{n}=\min \left(\gamma^{n-1}+\rho_{\nabla} \operatorname{det}\left(\mathbf{D u}^{n}\right), 0\right) .
\end{aligned}
$$

It is necessary to define the finite elements used for each rod and for the rectangular domain $\Omega$ included between these two rods (cf. Figure 4). Classically, the various rods are discretized by Hermite finite-elements with four degrees of freedom per node: the two components of 
the displacement field and the two components of its gradient with respect to the curvilinear abscissa. Domain $\Omega$ is discretized by specific finite elements: each rectangular element comprises four edges, with necessarily one edge on $\Gamma_{S}$ and another on $\Gamma_{N}$. For the fictitious domain

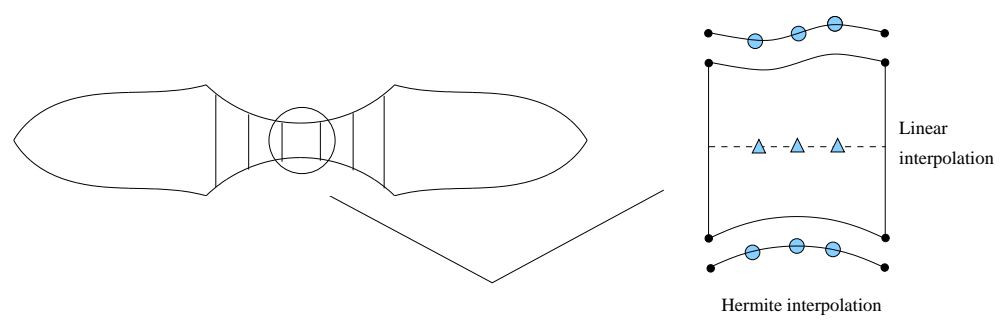

Figure 4. Fictitious domain and Gauss points where the multipliers are controlled: orientationpreserving condition (triangle) and inextensibility (circle).

(contact), the finite elements use a Hermite interpolation in the $s$ direction and a linear one according to $t$. The contact elements are thus rectangular with four nodes carrier of generalized displacements of the two rods. The shape of the lower and upper rods are thus of the curves of order three. More precisely, the control of the contact must be carried out on three Gauss points aligned in the direction $s$ located at $t=1 / 2$. In this way, we implemented a "symmetrical" description of the contact without privileging either of the two rods. With this method, one can plan to activate the elements only when the facets are close enough. The three multipliers associated with the inextensibility condition are updated and controlled in three Gauss points located on the rods.

As mentioned in $[2,3]$, the algorithm presented above fails to converge toward postbuckling shapes. Because the problem of the first step is uncoupled in terms of the vertical and horizontal components of the displacement, the algorithms tend to reach the trivial solution $(\mathbf{u}=\mathbf{0})$. In other words, the coupling between components is only performed by the inextensibility condition. To overcome this difficulty, we consider the iterative scheme, based on the DCalg1 algorithm, where the first step is performed by replacing the type II Lagrangian $\mathrm{L}_{\mathrm{II}}$ by the new functional, depending on the previous iteration involving a vanishing term,

$$
\mathrm{L}_{\mathbb{I}}{ }^{n}\left(\mathbf{v}, \boldsymbol{\tau}_{S}, \boldsymbol{\tau}_{N}, \boldsymbol{\tau}_{\nabla} ; \mu_{S}, \mu_{N}, \nu\right)=\mathrm{L}_{\mathbb{I}}\left(\mathbf{v}, \boldsymbol{\tau}_{S}, \boldsymbol{\tau}_{N}, \boldsymbol{\tau}_{\nabla} ; \mu_{S}, \mu_{N}, \nu\right)+\int_{0}^{\ell}\left(\mathbf{v}-\mathbf{u}^{n}\right) . \mathbf{A}\left(\mathbf{v}-\mathbf{u}^{n}\right) d s,
$$

where $\mathbf{A}=a\left(\begin{array}{ll}2 & 1 \\ 1 & 2\end{array}\right), a$ is a strictly positive coefficient, and $\mathbf{u}^{n}$ is the displacement obtained at the previous iteration $n$.

\section{Confined buckling}

In the first example we considered a flexible rod between two flat obstacles (cf. Figure 5a). This example was already studied in [3] using a classical formulation of the contact; a Lagrange multiplier was introduced, identified to the contact reaction and a Generalized Newton method was used to solve this problem. Here we introduced 18 one dimensional Hermite finite elements for the rod and 26 two-dimensional fictitious elements between the rod and the two obstacles 
(cf. Figure 5). Some confined buckling configurations may be determined according to different
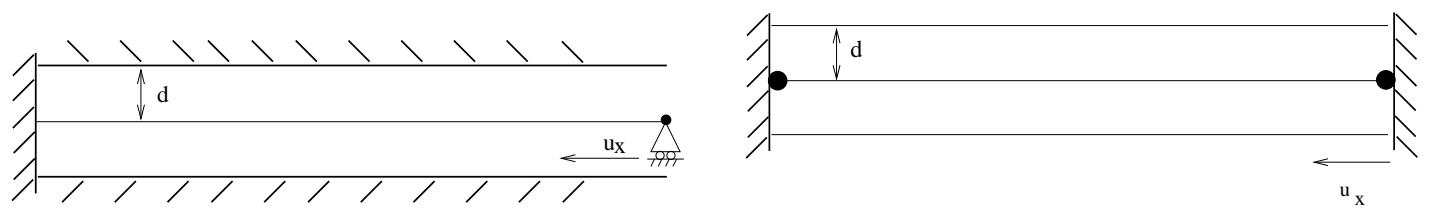

Figure 5. One flexible rod confined between two obstacles; 26 fictitious elements.

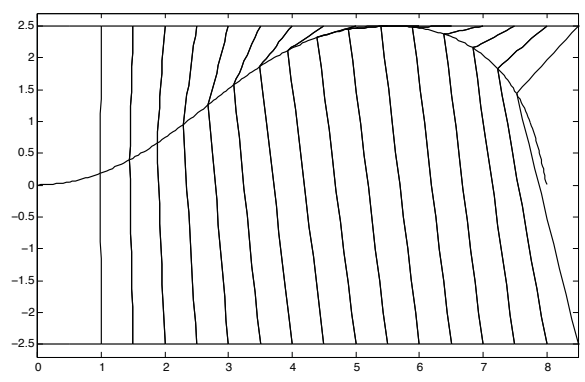

a)

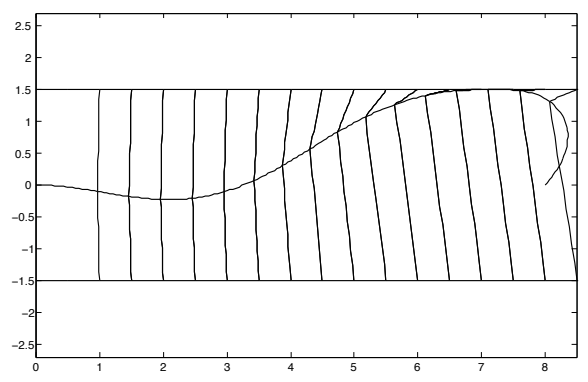

b)

Figure 6. Two confined buckling shapes (18 finite elements for the rod and 26 fictitious elements): a) $d=2.5 \mathrm{~m}$, grazing contact, b) $d=1.5 \mathrm{~m}, 1$ top element contact

values of the distance $d$. The values of $\ell$ and $E I$ were $: \ell=10 \mathrm{~m}, E I=1000 \mathrm{~N} / \mathrm{m} 2$. The convergence thresholds were $\varepsilon_{1}=10^{-7}$ and $\varepsilon_{2}=10^{-3}$; the parameters $\rho=150$ for DC algorithm and $a=1000$. Figure $5 \mathrm{a}$, shows a rod with a clamped origin and a simply supported end and the imposed displacement $U_{x}$ of the end of the rod is fixed to $2 \mathrm{~m}$. In Figure 6 two confined buckling shapes were plotted corresponding to two values of the gap $d$, with the fictitious domains.

Table II. Behaviour of the algorithm (Rod : 36 elements, 3x36 Gauss points; Fictitious : 4 elements, $3 * 4$ Gauss Points)

\begin{tabular}{l|cccc}
\hline Curve $/ \mathrm{d}(\mathrm{m})$ & $1) d=2.5$ & $2) d=2.1$ & $3) d=1.7$ & $4)$ \\
\hline Nb of iter DCAlg1 (internal loops) & $536(8)$ & $414(8)$ & $501(9)$ & $394(9)$ \\
Nb of Gauss points: $\mu=\mu^{+}$ & 49 & 48 & 36 & 28 \\
Nb of Gauss points: $\nu$ & 1 & 3 & 6 & 3 \\
\hline
\end{tabular}

With $d$ equal to $2.5 \mathrm{~m}$ only a grazing contact was associated with the first buckling branch. In this case, 49 inextensibility multipliers and one orientation-preserving multiplier were activated. Until $1.7 m$ the contact area increased to reach 2 fictitious elements (6 Gauss points). 


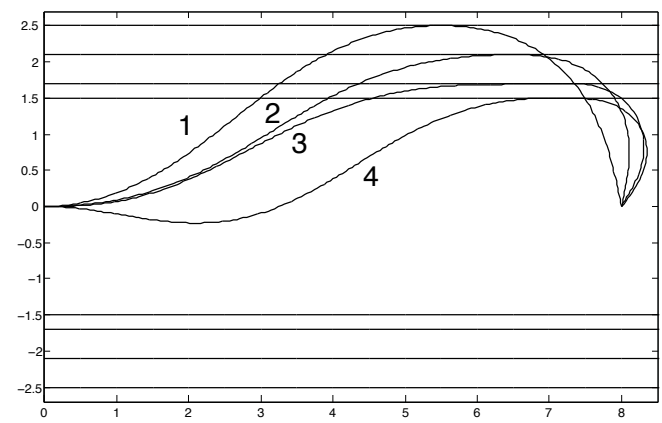

Figure 7. Some confined buckling shapes : a) curve $1(d=2.5 m$, grazing contact), curve $2(d=2.1 m$, 1 top element contact), curve $3(d=1.7 m, 2$ top element contacts), curve $4(d=1.5 m, 1$ top element contact).

At $1.5 \mathrm{~m}$, the rod jumped to a wave-like configuration with a single element point contact (3 Gauss Points). The active inextensibility multipliers decreased from 49 to 28 (cf. Figure 7).
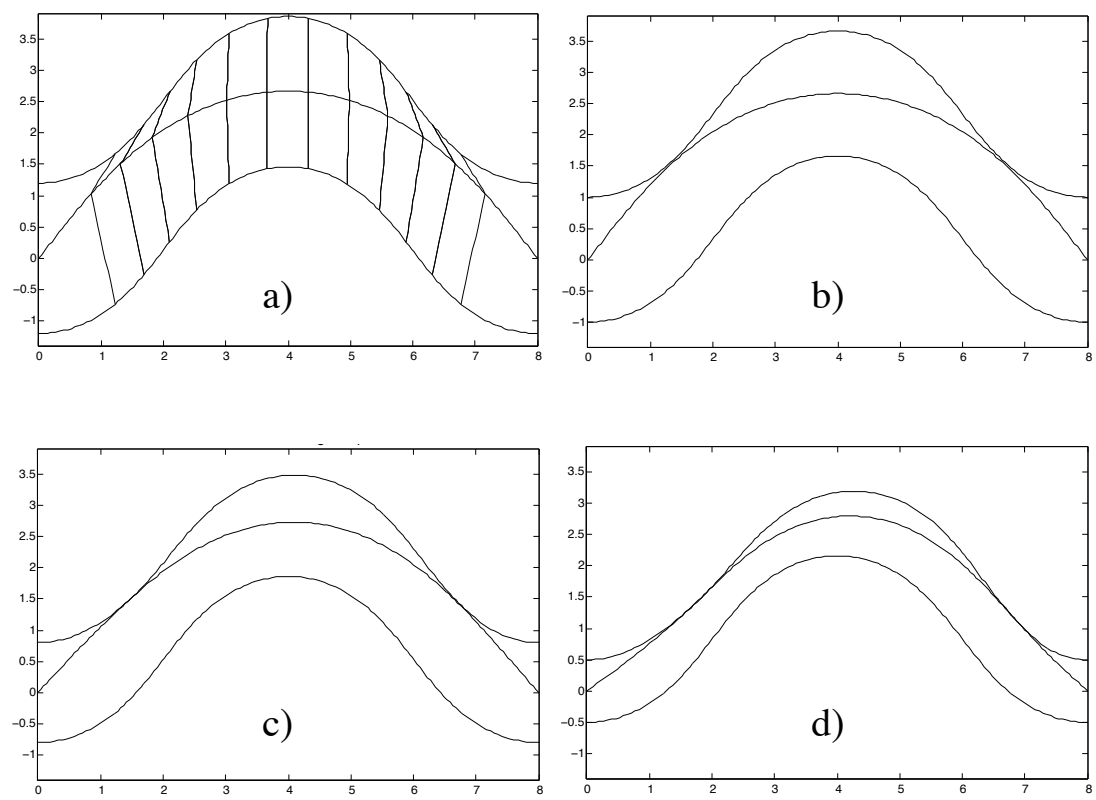

Figure 8. Three flexible rods; 22 fictitious elements are considered. Four configurations associated with different gaps: a) $d=2.4 m$; b) $d=2 m$; c) $d=1.6 m$; d) $d=1 m$;

The next example no longer considers obstacles but three flexible rods that can enter into contact with each other (cf. Figure 5b). Numerically the convergence of the algorithm is more difficult to achieve; different solutions can be obtained by considering different initial solutions

Int. J. Numer. Meth. Engng 2000; 00:1-6 
and oscillations between two solutions may appear. To obtain such a solution almost 500 iterates of DCAlg1 are needed. Some confined buckling configurations are plotted according to different values of the distance $d$ in Figure 8 . The contact zones occur in two different positions and increase when the value of $d$ decreases.

\section{Application to cellular media}

The behavior of a cellular medium is studied here, resulting from an assembly of rods. In fact, each unit cell of the network is made up of 6 rigidly connected rods (cf. Figure 9): the angle between two rods is assumed to be independent of the deformation [3]. Although these examples are geometrically more complex than the buckling of a single rod, presented in a previous section, the algorithm needs a regularization parameter $a$ smaller leading to a faster convergence. Indeed, the complexity of the structure with potential defects naturally leads towards a global buckling mode with no cycling between different modes. This value of $a$ was used in all the cellular samples. Recall that in non linear elasticity the homogenization procedure cannot provide an effective behaviour law for the honeycomb material starting from one unit cell as the elementary representative volume [10]. However to investigate the behavior of this structure in certain situations, an increasing number of cells were considered in a specimen. The aim was to capture the possible configuration paths accounting for eventual self contact. The studied case was a confined compression test consisting of a specimen pressed into a box on its upper side. For convenience the boundary conditions were approximated without taking into account unilateral contact conditions between the structure and the box. Only 6 Hermite finite elements were used for each rod and 4 fictitious elements were considered (cf. Figure 9). In this case, the number of iterations of DCAlg1 was lower than in the previous example; the inextensibility and the orientation-preserving multipliers, introducing non convexity, increased simultaneously. Furthermore, it is convenient to restrict to 5 the number of DC iterations (internal loops) without over-penalizing the Uzawa iterations (cf. Tables II and III).

Table III. Behaviour of the algorithm (Rod : 36 elements, 3x36 Gauss points; Fictitious : 4 elements, $3 * 4$ Gauss Points)

\begin{tabular}{l|cccccc}
\hline Imposed displacement $(\mathrm{m})$ & 4 & 4.5 & 4.6 & 4.7 & 4.8 & 4.9 \\
\hline Nb of iter DCAlg1 (internal loops) & $214(10)$ & $150(11)$ & $166(22)$ & $51(45)$ & $68(49)$ & $108(50)$ \\
Nb of iter DCAlg1 (5 internal loops) & $214(5)$ & $152(5)$ & $170(5)$ & $90(5)$ & $108(5)$ & $130(5)$ \\
Nb of Gauss points: $\mu=\mu^{+}$ & 21 & 33 & 48 & 54 & 53 & 55 \\
Nb of Gauss points: $\nu$ & 0 & 2 & 2 & 3 & 4 & 4 \\
\hline
\end{tabular}

In the next example, a cellular medium resulting from an assembly of 18 elementary cells was considered with the same boundary conditions. Only 5 Hermite finite elements and 3 fictitious elements were used for each rod and for each cell respectively (cf. Figure 10a). Until contact the configuration remained regular with repetition of the same pattern with a central crushed 

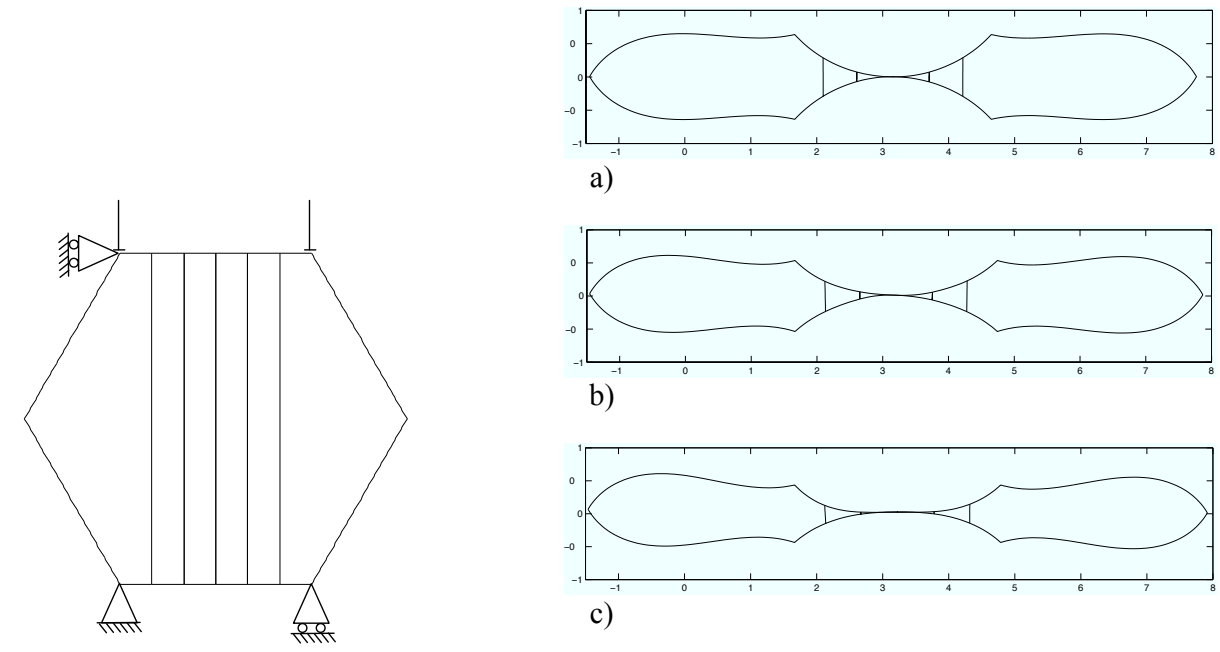

a)

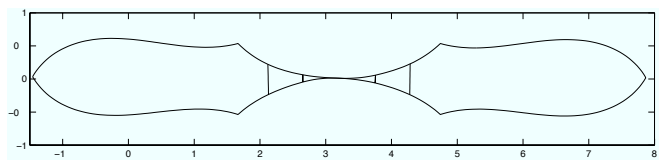

b)

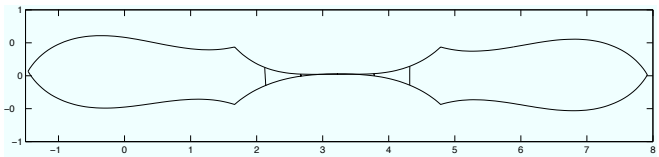

c)

Figure 9. One Cell with 36 elements and 4 fictitious elements are considered.

cell identical to the one presented in the previous simple example (cf. Figure 10b and Figure 11). When contact increases, distortion of the numerical solution appears foreshadowing shear band. This behavior was confirmed by more complex simulations with and without friction [6]. In a single step, a global displacement $U_{y}$ of the upper side of the box was imposed $\left(U_{y}=6 \mathrm{~m}\right.$ corresponding to $26 \%$ of the total height) requiring a large number of of DCAlg1 iterations to attain the buckling mode (cf. Table IV). Starting from this mode, contact did not modify it fundamentally and fewer iterations were needed to correct the configuration. As previously, the inextensibility and the orientation-preserving multipliers, introducing non convexity, increased simultaneously due to the confinement of the global structure (cf. Table IV).
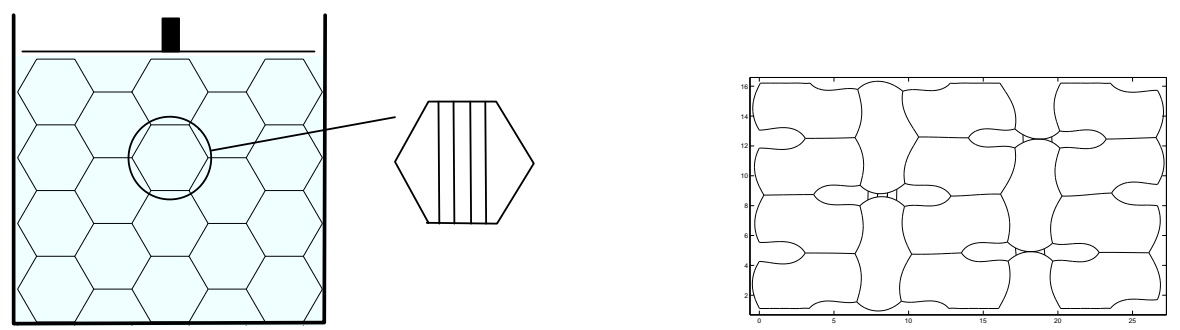

Figure 10. a) 18 cells with 355 Hermite finite elements and 54 fictitious elements are considered; b) confined buckling shape for $U_{y}=8 m$ ( $39 \%$ of the total height). 


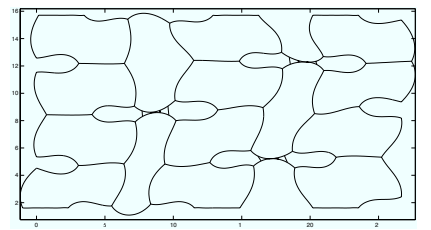

a)

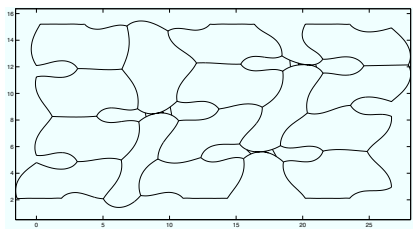

b)

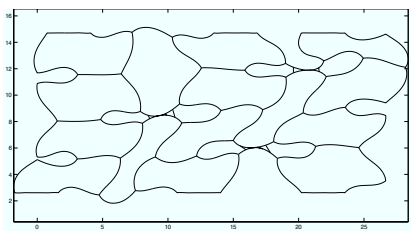

c)

Figure 11. Some confined buckling shapes : a) $U_{y}=9 \mathrm{~m}$ (39\% of the total height); b) $U_{y}=10 \mathrm{~m}(43 \%$ of the total height); c) $U_{y}=11 m$ (48\% of the total height).

Table IV. Behaviour of the algorithm (Rod : 355 elements, 3x355 Gauss points; Fictitious : 54 elements, $3 * 54$ Gauss Points)

\begin{tabular}{l|cccccccc}
\hline Imposed displacement $(\mathrm{m})$ & 6 & 8 & 8.5 & 9 & 9.5 & 10 & 10.5 & 11 \\
\hline Nb of iter DCAlg1 (5 internal loops) & 2000 & 1097 & 249 & 232 & 233 & 234 & 238 & 358 \\
Nb of Gauss points: $\mu=\mu^{+}$ & 355 & 455 & 458 & 469 & 474 & 485 & 480 & 498 \\
Nb of Gauss points: $\nu \neq 0$ & 0 & 2 & 3 & 3 & 4 & 5 & 5 & 6 \\
\hline
\end{tabular}

\section{Comments}

In this paper, we succeed in introducing the numerical treatment of contact and self-contact without perturbing the structure of a D.C. strategy initially dedicated to the buckling simulation of thin structures. This result is obtained using a relaxed orientation-preserving constraint in a fictitious finite element. The D.C. structure of the functional is only assured in bi-dimensional modeling and the three-dimensional case seems more difficult to achieve. The convergence of the algorithm is not penalized by the contact treatment; the inextensibility condition remains the main difficulty. From a practical point of view, in an incremental process it will probably be necessary to use adaptive remeshing to conserve initial non degenerated fictitious elements with opposite facets.

\section{REFERENCES}

1. P. Alart and A. Curnier. A mixed formulation for frictional contact problems prone to newton like solution methods. Comp. Meth. Appl. Mech. Eng., 92:353-375, 1991.

2. P. Alart and P. Pagano. Confined buckling of inextensible rods by convex difference algorithms. C.R. Mécanique, 330:819-824, 2002.

3. P. Alart and P. Pagano. DC solutions of postbuckling problems. J. of Global Optimization, 29:353-370, 2004.

4. G. Auchmuty. Duality algorithms for nonconvex variational principles. Num. Funct. An. and Opt., 10:211-264, 1989.

5. J.M. Ball. Global invertibility of Sobolev functions and the interpenetration of matter. Proc. Royal Soc. Edinburgh, 88A:315-328, 1981.

6. M. Barboteu, P. Alart, and S. Pagano. Modélisation de problèmes non linéaires de grande taille : grandes

Int. J. Numer. Meth. Engng 2000; 00:1-6 
déformations et autocontact dans un milieu cellulaire. Revue Européenne des Eléments finis, 11:447-461, 2002.

7. Ph. G. Ciarlet and J. Necas. Injectivity and self-contact in nonlinear elasticity. Arch. Rational Mech. Anal., 97 (3):171-188, 1987.

8. G. De Saxcé. Sur quelques problèmes de Mécanique des Solides considérés comme matériaux à potentiels convexes. PhD thesis, Université de l'Etat à Liège, Belgique, 1986.

9. R. Glowinski and P. Le Tallec. Augmented Lagrangian and operator-splitting methods in nonlinear mechanics. SIAM Studies in Applied Mathematics, Philadelphia, PA, 1989.

10. S. Müller. Homogenization of non convex integral functionals and cellular elastic materials. Arch. Ration. Mech. Anal., 99:189-212, 1987.

11. S. Pagano and P. Alart. Solid-solid phase transition modelling : relaxation procedures, configurational energies and thermomechanical behaviours. Int. J. of Eng. Sci., 37:1821-1840, 1999.

12. S. Pagano, P. Alart, and O. Maisonneuve. Solid-solid phase transition modelling. local and global minimizations of nonconvex and relaxed potentials. isothermal case for shape memory alloys. Int. J. of Eng. Sci., 36:1143-1172, 1998.

13. G.E. Stavroulakis and P.D. Panagiotopoulos. Convex multilevel decomposition algorithms for nonmonotone problems. Int. Journ. for Num. Meth. in Eng., 36:1945-1966, 1993.

14. P. Wriggers. Finite element algorithms for contact problems. Arch. Comput. Meth. Engrg., 2:1-49, 1995. 